DOCTRINA

\title{
Cumplimiento y ejecución de los acuerdos de transacción derivados de la mediación internacional a través de los contratos inteligentes
}

\author{
Enforceability and execution of transaction agreements derived \\ from international mediation through smart contracts
}

\author{
Wendolyne Nava González \\ Universidad Autónoma de Ciudad Juárez, México
}

Víctor Manuel Morales Rocha

Universidad Autónoma de Ciudad Juárez, México

\begin{abstract}
RESUMEN El objetivo de este trabajo fue explorar cómo los contratos inteligentes pueden ser una herramienta para facilitar y agilizar el cumplimiento y ejecución de los acuerdos de transacción comercial derivados de una mediación internacional. El criterio metodológico utilizado fue el análisis crítico del estado actual de la ejecución de acuerdos de transacción derivados de una mediación internacional y examina las particularidades de los contratos inteligentes en este respecto, para hacer una propuesta en específico. La conclusión a la que se llega es que, a pesar de los muchos retos que en la actualidad tienen los contratos inteligentes, sus características únicas, como la descentralización, autonomía, inmutabilidad y ejecución automática, hacen posible la reducción significativa del incumplimiento de los acuerdos de transacción derivados de una mediación internacional.
\end{abstract}

PALABRAS CLAVE Ejecución de acuerdos, contratos inteligentes, mediación internacional, blockchain.

ABSTRACT The objective of this work was to explore how smart contracts can be a tool to facilitate and speed up the enforceability and execution of commercial transaction agreements derived from international mediation. The methodology used was the evaluation of the current state of international mediation and examines the peculiarities of smart contracts. The conclusion reached is that despite the many challenges that smart contracts currently have, their unique characteristics, such as decentralization, autono- 
my, immutability and automatic execution, make it possible to significantly reduce noncompliance with transaction agreements. derived from international mediation.

KEYWORDS Execution, smart contracts, international mediation, blockchain.

\section{Planteamiento del problema}

Por regla general, existen tres opciones de solución de conflictos que emanan del comercio internacional tradicional o en línea: los tribunales estatales, los órganos supranacionales y los mecanismos extrajudiciales de resolución de conflictos o medios alternativos de solución de conflictos (MASC)1 (Pereznieto y Silva, 2007: 616). Siguiendo a Nuria González, la cual señala que «los medios alternos de solución de conflictos son herramientas coadyuvantes de la administración de justicia que complementan al sistema de justicia tradicional, con el objetivo de evitar de inicio la tensión generada en un proceso litigioso o procesos arbitrales buscando arreglos en pro de la convivencia pacífica» (González, 2014: 103), se puede entender por qué estos procedimientos son cada vez más populares: son más rápidos, más eficientes y sobre todo más amigables, ya que permiten a las partes dirimir sus controversias sin afectar la actividad comercial.

En la mediación internacional —en la cual se centra este artículo-, las partes eligen a una institución descentralizada que, a través de uno o varios terceros, las apoya para resolver su controversia mediante un arreglo amistoso, el cual se plasma en un acuerdo de transacción que firman los involucrados. Cuando la parte afectada se niega a cumplir con el acuerdo de transacción de forma voluntaria, la parte favorecida tiene dos opciones. La primera es solicitar la ejecución coactiva ante un órgano jurisdiccional, lo cual trae como resultado que todas las ventajas propias del procedimiento de mediación desaparezcan, porque las partes tendrán que enfrentarse en los tribunales, un procedimiento lento y costoso. La segunda, dadas las implicaciones del primer escenario, es no hacer nada.

De lo anterior se desprende la necesidad de encontrar una forma más eficiente de ejecutar acuerdos de transacción derivados de una mediación internacional, ya que la realidad indica que es un recurso no muy utilizado, en virtud de que no ha garantizado la eficacia sobre los acuerdos de transacción y las partes se han visto obligadas a acudir al arbitraje o tribunales ordinarios para hacer valer estos acuerdos, lo que aumenta los costos y en consecuencia desincentiva su uso (Tasende, 2020). En este sentido, un estudio del International Mediation Institute a operadores jurídicos sobre el tema señaló que «más del 90\% consideraba que la inexistencia de un mecanismo

1. En inglés: alternative dispute resolutions (ADR). 
internacional de ejecución constituía un impedimento para que se recurriera con más frecuencia a la mediación para resolver controversias transfronterizas» (CNUDMI, 2015).

Así, hoy vivimos en un mundo que se está desarrollando en un entorno digital, por lo que las categorías tradicionales de entendimiento del derecho se están modificando constantemente. El objetivo de este trabajo es explorar cómo la tecnología, y en particular los contratos inteligentes, pueden ayudar a solucionar uno de los retos más complejos que enfrenta la mediación internacional: el cumplimiento y ejecución de acuerdos de transacción comercial, a fin de brindar a las partes no solo mayor previsibilidad y certeza jurídica, sino además la posibilidad de que ahorren tiempo y dinero. Para cumplir este objetivo, este trabajo se divide en los siguientes apartados: primero, se describe el estado actual de la ejecución de acuerdos de transacción en la mediación internacional. En el segundo apartado, se analiza la utilización de los contratos inteligentes para garantizar el cumplimiento y ejecución de los acuerdos de transacción comercial derivados de la mediación internacional, donde se presenta una propuesta en específico. En la última parte, se explican los retos que esta propuesta presenta, con las conclusiones correspondientes.

\section{Estado actual de la ejecución de acuerdos de transacción en la mediación internacional}

La mediación es un procedimiento en el que las partes en un litigio solucionan una disputa de manera amistosa con la ayuda de un tercero. En términos generales, se puede decir que es un procedimiento que en la práctica está sujeto a pocas formalidades. No obstante, se debe basar en principios importantes, como la imparcialidad e independencia del tercero neutral, el trato igualitario a las partes, la confidencialidad, la voluntariedad, la flexibilidad, la legalidad y la economía (González, 2014: 24).

Existen en la doctrina numerosas definiciones de la mediación. Sin embargo, se considera que es en las fuentes primarias, en particular los instrumentos internacionales, donde se puede encontrar una definición más adecuada, ya que por lo general se elaboran teniendo en cuenta la diversidad internacional. Así, la Directiva 2008/52/ CE del Parlamento Europeo, del 21 de mayo de 2008, sobre ciertos aspectos de la mediación en asuntos civil y mercantiles, describe a la mediación de la siguiente manera: «Aquel proceso estructurado en el que dos o más partes en un litigio intentan voluntariamente alcanzar por sí mismas un acuerdo sobre resolución de su litigio con ayuda de un mediador».

Desde su aparición, este mecanismo podría haber sido usado con más frecuencia en el comercio internacional, en sustitución de los procesos ante tribunales, por diversas razones. Uno de los motivos más importantes es que permite la preservación de las relaciones comerciales, ya que el acuerdo lo construyen las partes, con ayuda de un 
tercero, basados en una dinámica de ganar-ganar, lo que facilita las operaciones comerciales internacionales y reduce en forma significativa los costos de impartición de justicia por parte del Estado. ${ }^{2}$ Aun así, la mediación no ha sido el mecanismo predilecto para resolver conflictos en el ámbito comercial internacional (González, 2020), por lo que en los últimos años se han buscado soluciones para ayudar a incentivar su uso.

A nivel internacional, existen varios instrumentos que regulan a la mediación, los cuales han fomentado la búsqueda de soluciones armonizadas que faciliten la utilización de este mecanismo y al mismo tiempo garanticen la salvaguarda de ciertos aspectos de derecho internacional privado (López Vallés y López Cárdenas, 2014: 8). La Comisión de las Naciones Unidas para el Derecho Mercantil Internacional (CNUDMI) ha jugado un papel fundamental en promover la armonización y unificación progresiva de este mecanismo de solución de conflictos comerciales de índole internacional. En este respecto, la Comisión ha señalado: «Muchos profesionales del derecho han adelantado el parecer de que la vía de la conciliación ganaría adeptos si se dotara al arreglo concertado en el curso de la conciliación de un régimen de ejecución ágil o de una fuerza ejecutoria idéntica o similar a la de un laudo arbitral» (CNUMDI, 2015: 6).

En un inicio, la CNUDMI expidió instrumentos utilizando el término conciliación; a partir de 2018, este término cambió a mediación. Dada su relevancia, a continuación se hará una descripción de estos esfuerzos, con especial énfasis en la ejecución de acuerdos derivados de este procedimiento, por ser la parte medular de este artículo.

\section{Reglamento de Conciliación de 1980}

El 4 de diciembre de 1980, la CNUDMI aprobó el Reglamento de Conciliación, un instrumento con reglas procesales de conciliación muy básicas. Sin embargo, destaca porque estableció directrices sobre ciertas cuestiones muy interesantes, como la admisión de pruebas en otros procedimientos, la confidencialidad, el derecho de las partes a acudir a la vía judicial o arbitral durante un procedimiento de conciliación, e incluso incluye una cláusula modelo de conciliación. No obstante, no regula ni hace mención al tema de ejecución del acuerdo de transacción derivado de la conciliación.

A partir de su publicación, este instrumento sirvió como modelo para instituciones que proveían servicios de mediación o conciliación en todo el mundo. Sobre la base del Reglamento, más adelante la CNUDMI propuso la creación de una ley modelo. ${ }^{3}$

2. Antonio M. Prida, «Convención de la ONU: Acuerdos producto de la mediación», El Semanario, 20 de julio de 2020 , disponible en bit.ly/2RmqBMn.

3. «Reglamento de Conciliación», Comisión de Naciones Unidas para el Derecho Mercantil Internacional, 1980, disponible en bit.ly/3fQsyKw. 


\section{Ley Modelo sobre Conciliación Comercial de 2002}

En su $35 .^{\circ}$ período de sesiones celebrado en Nueva York, del 17 al 28 junio de 2002, la CNUDMI aprobó la Ley Modelo sobre Conciliación Comercial, con su correspondiente guía para su incorporación al derecho interno y utilización. El objetivo de dicho instrumento fue promover esta vía alterna como método para solucionar conflictos de índole comercial internacional a fin de reducir costos, para fomentar un clima de cooperación entre las partes y prevenir futuros litigios. La idea original era que, a través de los años, este mecanismo se posicionara como una solución jurídica de armonización a nivel global en la materia, a fin de sentar las bases para la modernización del derecho interno de los Estados en este sentido.

Cabe señalar que una ley modelo solo contiene los rasgos mínimos de un instrumento legislativo, a fin de que cada país los adaptara atendiendo a las necesidades y particularidades específicas de su derecho procesal interno y facilitar su incorporación. Así, por ejemplo, los países podrían optar por que la Ley Modelo se aplicara no solo en el ámbito internacional, sino también en el nacional. Sin embargo, en términos generales, los Estados que la adoptaron hicieron cambios mínimos, apostando por la armonización a nivel mundial y lograr que el procedimiento fuera familiar para todos los actores internacionales que intervinieran (página 9 de la Ley Modelo).

La Ley Modelo estableció definiciones, principios, reglas procesales y directrices en relación con la conciliación internacional, basados en el Reglamento de Conciliación de 1980. En relación con el tema medular de este artículo, el acuerdo de transacción y su ejecución, la Ley Modelo estableció en su artículo 14:

Si las partes llegan a un acuerdo por el que se resuelva la controversia, dicho acuerdo será vinculante y susceptible de ejecución [...] los Estados promulgantes podrán incorporar una descripción del medio de ejecución de los acuerdos de transacción o remitirse a las disposiciones que rijan su ejecutoriedad.

Lo primero que destaca es que el instrumento señaló con claridad que el acuerdo será «vinculante y susceptible de ejecución», lo cual en principio fue un gran acierto en virtud de que estas dos características dotaron a la Conciliación Internacional de un poder de ejecución similar al del laudo arbitral. Lo segundo es que - contrario al objetivo del instrumento, que era la armonización- no estableció ningún tipo de condiciones generales para garantizar su ejecución, sino que dejó abierta la puerta para que cada Estado aplicara sus propias reglas al respecto, ya que, según se desprende de los documentos oficiales de la CNUDMI, hay grandes diferencias entre los Estados sobre los métodos de ejecución, por lo que fue imposible llegar a un consenso (página 55 de la Ley Modelo).

La falta de armonización en el tema de ejecución de los acuerdos de transacción comercial derivado trajo como resultado que, a la fecha de publicación de este artícu- 
lo, y según lo establece la Comisión de las Naciones Unidas para el Derecho Mercantil Internacional, solo 33 Estados han implementado una legislación basada en la Ley Modelo, entre los cuales no está Chile. ${ }^{4}$

\section{Ley Modelo sobre Mediación Comercial Internacional y Acuerdos de Transacción Internacionales resultantes de la Mediación 2018}

A raíz de lo anterior, el 20 de diciembre de 2018 se modificó la Ley Modelo sobre Conciliación Comercial Internacional, de 2002, para transformarse en la Ley Modelo sobre Mediación Comercial Internacional y Acuerdos de Transacción Internacionales Resultantes de la Mediación, de 2018.

Como indica el nombre del instrumento, la reforma se traduce básicamente en dos grandes cambios. El primero fue que se adoptó el término mediación, en sustitución de conciliación, pues la Ley Modelo de Conciliación de 2002 no hizo distinción entre estos términos, sino que los englobó en uno mismo y sumó cualquier otro que pudiera ser equivalente. Si bien es cierto que la doctrina establece distinciones entre dichos procedimientos tomando en consideración diversos criterios, también lo es que la Comisión consideró que, para fines pragmáticos y especialmente legislativos, no era necesario hacerlo, pues el instrumento se podría aplicar para cualquier procedimiento voluntario en el que interviene un tercero para apoyar a las partes a solucionar un conflicto de una forma amigable, a fin de que pudiera ser adoptado por el mayor número de legislaciones. Sin embargo, ahora se consideró que el término mediación es una terminología más utilizada en la legislación interna de los Estados para referirse a procedimientos extrajudiciales de solución de conflictos en que interviene un tercero neutral para ayudar a las partes a llegar a un acuerdo amistoso; además, presume que le da muchísima más visibilidad al mecanismo (Strong, 2014).

El segundo y más importante cambio es que sumó un capítulo 3: «Acuerdos de transacción internacionales», que desarrolla más el tema de ejecución y en el que evidentemente se incorporaron los principios de la Convención de Singapur de 2018, de la cual se hablará más adelante. Este capítulo fue diseñado para poder ser agregado por los Estados a su derecho interno, de modo de eliminar las grandes diferencias que en la actualidad existen en relación con la ejecución de este tipo de acuerdos (página 2).

El capítulo 3 de la renovada Ley Modelo señala que este se aplicará «a los acuerdos internacionales resultantes de la mediación que hayan sido celebrados por escrito por las partes con el fin de resolver una controversia comercial» (artículo 16). Excluye los

4. Para más información, véase «Situación actual Ley Modelo de la CNUDMI sobre Mediación Comercial Internacional y Acuerdos de Transacción Internacionales Resultantes de la Mediación, de 2018 (por la que se modifica la Ley Modelo de la CNUDMI sobre Conciliación Comercial Internacional)», Comisión de las Naciones Unidas para el Derecho Mercantil Internacional, disponible en bit.ly/3vUs8s2. 
acuerdos relacionados con el ámbito familiar, de sucesiones o laborales. Asimismo, deja de lado todos los acuerdos aprobados por un órgano judicial o que puedan ejecutarse, como una sentencia de ese órgano judicial, y aquellos que se hayan transformado en un laudo arbitral ejecutable. El capítulo también señala claramente cuándo un acuerdo de transacción deberá considerarse internacional.

$\mathrm{El}$ artículo 18 del instrumento establece como principio general que para ejecutar los acuerdos de transacción se tomarán en cuenta las reglas procesales de los Estados y los del capítulo en comento, estableciendo requisitos que se deberán presentar a la autoridad competente, las cuales se pueden resumir en:

- Acuerdo de transacción firmado por las partes, requisito que se da por cumplido respecto a las comunicaciones electrónicas.

- Pruebas de que se llegó a un acuerdo de transacción como resultado de una mediación, como la firma del mediador, la cual puede constar en el acuerdo de transacción o en cualquier otro documento que haga constar dicho hecho.

- Las traducciones al idioma que sean necesarias.

Por último, el capítulo establece una lista exhaustiva de razones por las que una autoridad puede negar la ejecución de un acuerdo de transacción y las medidas que deben tomar en caso de recibir una reclamación relacionada.

El capítulo, ante una evidente falta de técnica legislativa, está redactado de una forma confusa y poco ordenada, lo que dificulta su lectura y comprensión. Sería recomendable que los Estados que incorporen dicho capítulo a su legislación interna hagan modificaciones en su redacción para facilitar el entendimiento y sobre todo su aplicación. Sin embargo, es innegable que esta adición a la Ley Modelo constituye un paso para fomentar la armonización sobre las condiciones mínimas necesarias para la ejecutabilidad de los acuerdos de transacción comerciales derivados de una mediación o conciliación internacional y evitar que sean inválidos con arreglo al derecho sustantivo aplicable. Además, sin duda abre la puerta a los acuerdos de transacción celebrados a través de medios electrónicos o digitales, los cuales seguramente se verán en aumento en los próximos años, dados los cambios radicales que se tendrán que hacer a raíz de la terrible pandemia que se sufre en la actualidad.

\section{La Convención de Naciones Unidas sobre los Acuerdos de Transacción Internacional Resultantes de la Mediación, 2018 (Convención de Singapur)}

Aunado a la modificación a la Ley Modelo de Conciliación Internacional de 2002 (ahora Ley Modelo de Mediación Internacional), el 20 de diciembre de 2018, la Asamblea General aprobó la Convención de las Naciones Unidas sobre los Acuerdos de Transacción Internacionales resultantes de la Mediación. La idea fue impulsar 
más esta figura a través de una Convención, en la que los países que la ratificaran se comprometieran a cumplir dichas disposiciones, a pesar de las grandes diferencias en la materia. Este marco jurídico armonizado, para el efectivo cumplimiento y ejecutabilidad en el orden interno y en el ámbito transfronterizo de los acuerdos de transacción, tiene la esencia de la exitosa Convención sobre el Reconocimiento y la Ejecución de las Sentencias Arbitrales Extranjeras, de 1958, la cual ha sido suscrita por 164 países. La ceremonia de apertura a la firma de la Convención se llevó a cabo el 7 de agosto de 2019 en Singapur. A julio de 2020, 53 países han firmado la Convención, pero solo seis la han ratificado: Arabia Saudita, Bielorrusia, Fiyi, Qatar, Sierra Leona y Singapur.

La Convención consta de dieciséis artículos, de los cuales los primeros nueve son equivalentes al capítulo 3 de la Ley Modelo, aunque redactados de una forma más clara. En términos generales, se espera que este instrumento logre:

- Que los Estados tengan un marco claro y uniforme para facilitar la ejecución de los acuerdos de transacción comercial internacional en jurisdicciones diferentes.

- Lograr el efectivo y rápido cumplimiento y ejecutabilidad, en el orden interno y en el ámbito transfronterizo, de un acuerdo de transacción comercial internacional.

- Evitar que las partes de una controversia mercantil internacional que se someta a un procedimiento de mediación o conciliación se vean afectadas por un régimen jurídico que impida la ejecución del acuerdo de transacción resultante.

- Eliminar la práctica a través de la cual una controversia que se resuelve mediante conciliación o mediación se remita después al arbitraje, al solo efecto que se pronuncie un laudo consentido.

De este análisis se desprende que la ejecución de los acuerdos de transacción establece un sistema mixto, es decir, estará regulada tanto por la lex fori como por la Convención de Singapur, siguiendo el criterio de Jaboco Balduini, que señaló: «En materia de procedimientos [...] el juez debe aplicar su propia ley (ad litem decidendam), será aplicable la ley del lugar donde el contrato se hubiere celebrado» (Tasende, 2020). Esto traerá como resultado que el procedimiento cambie de país de país y no haya una completa armonización.

A pesar de estos esfuerzos por parte de la CNUDMI, lo cierto es que, al final, el acuerdo de transacción comercial derivado de un proceso de mediación tiene su fundamento en la voluntad de las partes. Todo régimen de ejecución deberá respetar los límites del acuerdo entre las partes, incluyendo la elección del foro, el cual no siempre será favorable, en tanto esta Convención no sea firmada y ratificada en un considerable número de países alrededor del mundo. Hasta que eso suceda, el 
derecho internacional privado debería tomar en consideración otras opciones, complementarias a las tradicionales, que favorezcan una ejecución fácil y rápida, como el uso de la tecnología.

\section{Blockchain y contratos inteligentes}

\section{Cadena de bloques}

A modo de introducción, es importante explicar qué es la tecnología de cadena de bloques o blockchain y por qué puede ser utilizada para la creación y ejecución de contratos inteligentes. A partir de diversas definiciones, incluidas las de Nofer y otros (2017) y Swan (2015), entre otras, se puede describir una blockchain como una base de datos en la que, similar a un libro contable, se lleva un registro de cierto número de transacciones, las que forman un bloque inicial llamado génesis. Este se encadena con el segundo bloque, este a su vez con el tercero y así sucesivamente, lo que forma una cadena de bloques. Los bloques se replican en cada uno de los nodos que componen la red de la blockchain una vez que han sido validados. Esta validación de los bloques y sus transacciones se produce a través de un mecanismo de consenso entre los nodos, lo cual nos lleva al concepto de descentralización, es decir, que bajo este esquema no existe una autoridad central, como un banco, Gobierno u otro tipo de institución que almacena, administra y valida los registros.

Bajo este principio de descentralización, y con la conjunción de una variedad de tecnologías ya existentes, nace la moneda virtual llamada Bitcoin, la cual permite realizar transacciones monetarias entre pares (peer-to-peer) sin la necesidad de una autoridad central que emita la moneda y avale las transacciones. La validación, en cambio, la llevan a cabo los nodos o computadoras que conforman la red, y consisten en la verificación de funciones criptográficas de clave pública, entre las que se incluyen la firma digital de la transacción (Champagne, 2014). Una vez que se completa y valida un bloque de tamaño determinado, se crea un nuevo bloque, el cual será encadenado al anterior. Dicho encadenamiento también se produce a través de una función criptográfica, conocida como función hash (Champagne, 2014). ${ }^{5} \mathrm{El}$ proceso de encadenamiento de bloques se muestra en la figura 1. La firma digital de cada transacción de los usuarios de la plataforma, junto con el valor hash de cada bloque, garantizan la inmutabilidad de la cadena de bloques, ya que, de presentarse cualquier modificación a los registros almacenados, por mínima que esta sea, sería fácilmente detectable.

5. Una función hash es una operación matemática unidireccional que toma como entrada una cadena de caracteres de longitud variable y obtiene como salida una cadena de longitud fija, generalmente de cientos de bits. Dicha cadena resultante, o valor hash, es única, es decir, no puede haber dos cadenas de entrada que den como resultado el mismo valor hash. 


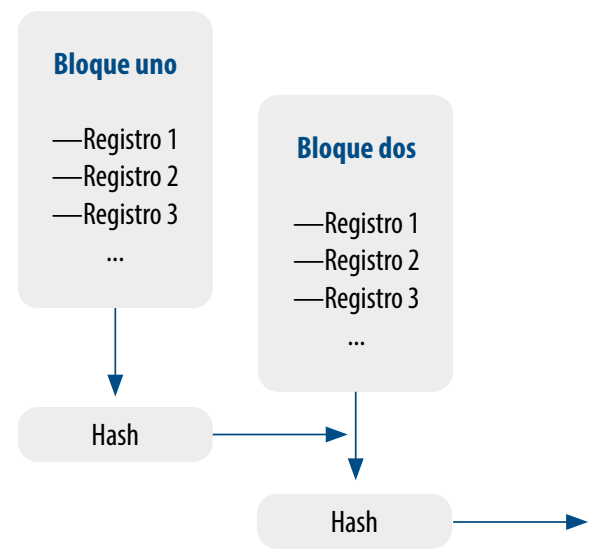

Figura 1. Esquema del encadenamiento de bloques

A manera de resumen, la tecnología de blockchain tiene cuatro características principales:

- Inmutabilidad: Una blockchain es un registro permanente de transacciones. Una vez que un bloque es añadido, este no puede ser alterado, lo cual genera confianza en las transacciones registradas.

- Descentralización: La blockchain es descentralizada, ya que no hay una autoridad central que tenga control sobre ella. Por otro lado, en su infraestructura no hay un punto central de falla, ya que cada nodo almacena una copia de los bloques. ${ }^{6}$

- Consenso: Cada bloque es validado y agregado a la blockchain a través de un mecanismo de consenso entre los nodos que provee las reglas de validación. En la blockchain de Bitcoin, por ejemplo, este mecanismo de validación incluye una prueba de trabajo (proof of work). ${ }^{7}$

- Transparencia: Al ser la blockchain una base de datos abierta, cualquiera puede acceder y auditar las transacciones (Sultan, Ruhi y Lakhani, 2016: 49-57).

En las blockchain que manejan una moneda virtual, como Bitcoin o Ethereum, las transacciones son de acceso público, ya que ese modelo es el que conviene a dichas plataformas para la expansión en el uso de la criptodivisa. Sin embargo, los registros de transacciones de una blockchain no necesariamente tienen que ser públicos. Exis-

6. Vitalik Buterin, «Ethereum white paper», Ethereum, 9 de febrero de 2021, disponible en ethereum. org/en/whitepaper/.

7. Satoshi Nakamoto, «Bitcoin: A peer-to-peer electronic cash system», Bitcoin.org, disponible en bitcoin.org/bitcoin.pdf. 
ten entornos en que resulta más conveniente limitar el acceso a las transacciones a un conjunto específico de usuarios. Bajo esta premisa es que han surgido las blockchain privadas. En estas, la característica de transparencia ya descrita se mantiene, pero está restringida a los usuarios legítimos de la blockchain en cuestión. Asimismo, el mecanismo de consenso para validar las transacciones lo define la propia blockchain. Así, en el contexto de contratos inteligentes, se puede elegir entre una blockchain pública o una privada, de acuerdo con los tipos de contrato y sus necesidades de transparencia y privacidad.

\section{Contratos inteligentes}

El origen teórico de los contratos inteligentes lo encontramos en un artículo publicado por Nick Szabo en 1996, que los define como: «Un conjunto de promesas declaradas de forma digital, utilizando protocolos a través de los cuales las partes cumplen y verifican dichas promesas». ${ }^{8}$ Sin embargo, cabe destacar que en la década de los noventa no existía la tecnología para crear este tipo de contratos, por lo que la idea no se implementó en la práctica. No fue hasta el 2009, ${ }^{9}$ cuando apareció la versión 0.1 de Bitcoin en Sourceforge ${ }^{10}$ utilizando blockchains, que se abrió la puerta para que los contratos inteligentes se volvieran una realidad. Así, en 2013, Vitalik Buterin publicó un artículo en virtud del cual describe una nueva plataforma de código abierto (open source) llamada Ethereum, basada en la tecnología de blockchain, «con un lenguaje de programación integrado Turing completo, que puede ser utilizado para crear contratos». ${ }^{11}$ El desarrollo e implementación de Ethereum en 2014 trajo como resultado el nacimiento de los contratos inteligentes (smart contracts) en el mundo virtual, gracias a que esta cadena de bloques permite establecer comandos y programación, lo que abre la posibilidad de definir una serie de reglas o cláusulas específicas para un contrato.

En principio, un contrato es un acuerdo de voluntades que crea o transmite derechos y obligaciones a las partes que lo suscriben. Julien Bonnecase señala que

el contrato es una manifestación exterior de la voluntad, bilateral o unilateral, cuyo fin es el de engendrar; fundándose en una regla del derecho, en contra o en provecho de una o varias personas, un estado, es decir, una situación jurídica general y per-

8. Nick Szabo, «Smart contracts: Building blocks for digital markets», Phonetic Science, disponible en bit.ly/2S3SQA1.

9. Derivado del artículo mundialmente famoso que publicó Satoshi Nakamoto en 2008, titulado «Bitcoin: A peer-to-peer electronic cash system», disponible en bitcoin.org/bitcoin.pdf.

10. Sourceforge es un sitio web de colaboración para proyectos de software, disponible en sourceforge. net.

11. Buterin, «Ethereum white paper». 
manente, o al contrario, un efecto de derecho limitado que conduce a la formación, a la modificación a la extinción de una relación de derecho (Bonnecase, 1945: 99).

Tradicionalmente estos acuerdos se hacen de forma verbal o por escrito. Sin embargo, con la aparición de las TIC, la formación e incluso su ejecución ha ido evolucionando (Castrillón Víctor y Anahiby Becerril, 2015), hasta llegar a lo que ahora conocemos como contratos inteligentes.

En 2018, la Comisión Europea impulsó la creación del Observatorio Europeo de Blockchain, que al año siguiente emitió un informe titulado «Legal and regulatory framework of blockchains and smart contracts». En el reporte se definen a los contratos inteligentes como «un programa de computadora que se almacena en una cadena de bloques, al que una o más partes pueden acceder. Estos programas a menudo son autoejecutables y hacen uso de propiedades de la cadena de bloques como inmutabilidad, descentralización, etcétera» (Observatorio Europeo de Blockchain, 2019: 22). En otras palabras, los contratos inteligentes son programas para llevar a cabo una o varias tareas específicas y determinadas, de acuerdo con las instrucciones introducidas por un programador. Originalmente existen seis fases en la creación de un contrato inteligente:

- Las partes manifiestan su voluntad para alcanzar un acuerdo.

- Los contratantes especifican las condiciones del contrato.

- Un programador escribe el código informático que permitirá la ejecución automática cuando sucedan los hechos previstos.

- Cada una de las cláusulas se cifra mediante sistemas de autenticación y verificación, en una cadena de bloques.

- Las cláusulas registradas en el bloque se verifican por el sistema de consenso, con lo que se ejecuta en forma automática la prestación.

- Ejecutada la prestación, todos los nodos del sistema la reconocen como tal, haciéndola inalterable en la cadena de bloques (Smart Contracts Alliance, 2016: 12).

La implementación de contratos inteligentes requiere dos tipos de plataformas: una denominada smart contract plataform (SCP), la cual es la infraestructura que permite crearlos y operarlos sobre una blockchain; y otra llamada smart contract management system (SCMS), la cual es la interfaz gráfica amigable que permite a cualquier persona el uso y manejo de estos contratos (Bourque y Tsui, 2014: 11).

Ahora bien, el desarrollo de plataformas de contratos inteligentes no es una tarea fácil, en especial si se parte desde la creación de blockchain sobre la cual operarán. Sin embargo, existen herramientas que facilitan su desarrollo y operación. Un ejem- 
plo de ello es Ethereum, una blockchain que permite a cualquiera escribir contratos inteligentes y aplicaciones descentralizadas en que puedan crear sus propias reglas arbitrarias de propiedad, formatos de transacción y funciones de transición de estado (Buterin, 2015a). A pesar de ser una blockchain pública, su tecnología hace posible crear, a partir de esta, una blockchain de carácter privado. Ethereum permite generar los contratos inteligentes a través de un lenguaje de programación propietario llamado Solidity, además de almacenar datos y transacciones con Ether, la moneda asociada a la plataforma.

Existen también varias plataformas relacionadas con los contratos inteligentes:

- Counterparty: Plataforma para desarrollo y ejecución de contratos inteligentes y otro tipo de aplicaciones descentralizadas. Basada en la blockchain de Bitcoin, utiliza el mismo lenguaje de programación de Ethereum, Solidity.12

- Ganache: Es un entorno de desarrollo para la creación y ejecución de contratos inteligentes sobre la red de Ethereum. Este entorno facilita la creación de una blockchain privada y la conexión entre la blockchain y los contratos inteligentes que se desea ejecutar. ${ }^{13}$

En este punto resulta interesante aclarar que los contratos inteligentes en realidad no son inteligentes, pues no tienen la capacidad de aprender, lo cual es una de las particularidades de la inteligencia artificial. Sin embargo, tienen otras características que los hacen únicos, muchas de las cuales son derivadas de la cadena de bloques en la que estos se almacenan:

- Autoejecutables: Si el evento A sucede, entonces la consecuencia B se pondrá en marcha de forma automática.

- Inmutables: No pueden ser modificados o borrados.

- Autoverificables: Su cumplimiento no está sujeto a la interpretación de ninguna de las partes.

- Descentralizados: No requieren de intermediarios (abogados, notarios, jueces, etcétera) para su ejecución.

- Incorruptibles: Resulta prácticamente imposible hacer algún cambio o alteración.

- Transparentes: Todas las operaciones y la información están siempre visibles.

Estas características hacen que los contratos sean autónomos, seguros, resilientes y confiables, por lo que podrían ser utilizados en una diversidad de áreas o ámbitos.

12. Sitio web de The Counterparty Platform, disponible en counterparty.io/platform.

13. Para más información, véase «Ganache overview», Truffle Suite, disponible en bit.ly/3vVrz17. 
En la actualidad se cuenta con algunas plataformas para crear y ejecutar contratos inteligentes. Entre ellas destaca OpenLaw, ${ }^{14}$ la cual es un protocolo basado en la tecnología de blockchain para la creación y ejecución de acuerdos legales inteligentes. A través del protocolo, los abogados pueden firmar y almacenar digitalmente contratos inteligentes de una manera sencilla y sin necesidad de trabajar en forma directa con un programador.

Por último, para que el contrato inteligente pueda ejecutarse, es necesario que se cumplan ciertas condiciones, ya que funcionan bajo la lógica de si esto pasa, entonces esto sucede (if-then), que por lo general es información que está en la cadena de bloques o proviene del exterior o del mundo real y que debe transmitirse a la blockchain a través de un oráculo. Estos se han definido como «programas, empresas o incluso personas físicas que transmiten información del mundo real a la blockchain para que los contratos inteligentes puedan ejecutarse» (De Filippi y Wright 2018: 75). En caso de que el contrato inteligente necesite información externa, las partes deben designar al oráculo que se encargará de proporcionarla de manera confiable, a fin de que el contrato se verifique y autoejecute. Así, por ejemplo, un testamento inteligente podría ejecutarse en cuanto se emita el acta de defunción correspondiente (oráculo) y asignar los bienes a los herederos de forma automática. Otro ejemplo sería un contrato de arrendamiento inteligente, en que el arrendatario contaría con una tarjeta electrónica para acceder a su vivienda, la cual se programa para que, en cuanto el arrendatario incumpla con el pago, deje de funcionar en forma automática.

\section{Aplicación de los contratos inteligentes en la ejecución de acuerdos de transacción comercial derivados de la mediación internacional}

Como ya se mencionó, la implementación de los contratos inteligentes a nivel global todavía es muy limitada, debido, en parte, a que el modelo actual se restringe a ciertas funcionalidades y porque, desde el punto de vista legal, se encuentran todavía en una zona gris. En virtud de que la tecnología es relativamente nueva, en muchos de los casos los usos son pruebas piloto o pruebas de conceptos. Algunas áreas o ámbitos en los que ya se utilizan o se proyectan utilizar son:

- Sector financiero: El uso más común en este sector es la programación de pagos o compras automáticas de acciones, criptomonedas, etcétera.

- Ámbito inmobiliario: En este ámbito se utilizan para registro y cambio de propiedad, así como para la compra de propiedades.

14. Para más información, véase «Introducing OpenLaw», Consensys, 25 de julio de 2017, disponible en bit.ly/2RoV3p8. 
- Propiedad intelectual: Para el pago de regalías, particularmente útiles cuando los derechos de autor son compartidos.

- Ámbito energético: Para el pago y suministro de electricidad.

Los contratos inteligentes también se están utilizando para la solución de conflictos. Un ejemplo es Kleros, una plataforma de resolución de disputas descentralizada que fue programada en Ethereum tomando como base a los contratos inteligentes. La finalidad de Kleros es arbitrar todo tipo de conflictos, en especial los de índole internacional y escasa cuantía, a través de árbitros anónimos que son seleccionados al azar mediante el uso de tokens. ${ }^{15}$ Dadas las características de los contratos inteligentes, el procedimiento es rápido, barato, confiable, descentralizado y las resoluciones se ejecutan de forma automática. ${ }^{16}$

Estos contratos inteligentes también se podrían utilizar exclusivamente para la ejecución de los acuerdos comerciales derivados de procedimientos extrajudiciales de solución de controversias, en particular de la mediación internacional. La propuesta se presenta a continuación.

Con este panorama en mente, imaginemos que el acuerdo de transacción al que llegaron las partes, derivado de una mediación comercial internacional, se plasma en un contrato inteligente, es decir, en un programa informático que se aloja en una cadena de bloques. En virtud de que el contrato se encuentra en un lenguaje de programación (contractware), se hará necesario que este se ligue a un acuerdo preexistente, escrito en un lenguaje natural que las partes puedan leer, entender y firmar (Padilla Sánchez, 2020: 194). Lo anterior, para garantizar que los términos del acuerdo son válidos de acuerdo con la ley aplicable. En este sentido, primero se tendría que ejecutar el acuerdo en lenguaje natural; una vez que ha sido firmado por los involucrados, deberá ser cambiado a un lenguaje de programación que refleje de manera íntegra la voluntad de las partes. Esto no será tarea fácil, pues el contrato inteligente se verá obligado a contemplar todos los posibles escenarios que puedan afectar su desarrollo, ya que una vez que se aloja en la cadena de bloques no se puede cambiar, debido a que estos contratos son rígidos y deterministas.

También se deberá tomar en cuenta que, en principio, no todos los acuerdos de transacción se podrán ejecutar a través de un contrato inteligente, ya que algunas de las obligaciones que se pactan en estos convenios son bastantes complejas. Tal vez se haga necesario que algunas obligaciones de un mismo acuerdo se ejecuten a través de estos contratos y otras de forma tradicional.

15. Token, en el ecosistema de blockchain, es cualquier activo que sea transferible digitalmente entre personas. Estos tokens se emiten en una cadena de bloques, con mayor frecuencia en Ethereum. Dependiendo de las propiedades que se les asignen, tienen diferentes clasificaciones y usos.

16. Clément Lesaege, Federico Ast y William George, «Kleros: Short paper v1.07», Kleros, septiembre de 2019, disponible en kleros.io/whitepaper.pdf. 
Lo cierto es que, de adoptarse esta propuesta, los acuerdos de manera paulatina tendrían que cambiar su redacción y diseñarse en términos binarios para evitar o reducir al mínimo los errores en su programación. Es decir, el lenguaje debe ser condicional («si esto pasa, entonces...») o en términos de falso o verdadero. Para esto, los operadores jurídicos deberán estar mínimamente relacionados con el lenguaje del código en que estos contratos se escribirán, para poder trabajar de manera conjunta con los programadores, que no tienen formación jurídica.

En relación con el tema de los oráculos, como ya se apuntó, en principio los contratos inteligentes ejecutan de forma automática obligaciones a través de un código computacional, sin intervención del juicio humano (Sklaroff, 2017: 279). Sin embargo, en la mayoría de los casos es necesaria la información del exterior, a fin de ejecutar las condiciones contenidas a través de oráculos. A veces esa información es pública y de fácil acceso, como la que se encuentra en la Bolsa de Valores, pero en otras no. Supongamos que en el acuerdo de transacción comercial derivado de una mediación internacional se acordó que, una vez que la mercancía regrese a su lugar de origen, en condiciones óptimas para volver a ser puesto a la venta en cierta fecha preestablecida, el vendedor entregará al comprador una suma de dinero. Esta información no es pública y requiere una valoración para determinar que se cumplen con las condiciones establecidas en el acuerdo. En este caso, el oráculo no puede ser un programa ni una organización. Deberá ser forzosamente un ser humano, un tercero que proporcione esta información a la blockchain, a fin de que se ejecute el contrato y se transfiera el dinero al vendedor.

En este caso concreto, las partes deberán seleccionar quién será el oráculo, y aceptar la información que proporcione dicho tercero. Como manifiesta Padilla Sánchez (2020: 197), los oráculos humanos sacrifican la independencia de los contratos inteligentes frente al mundo exterior, ya que el juicio humano se vuelve un catalizador de su ejecución. Sin embargo, a medida que tecnologías como el internet de las cosas avancen, tal vez se pueda eliminar el factor humano por completo. Se propone que el tercero sea la institución que llevó a cabo la mediación internacional, una vez que reciba las pruebas que considere pertinentes, lo cual deberá constar en el acuerdo de transacción. Asimismo, debería brindarse acceso a tribunales para que intervenga en caso de ser necesario.

En los términos de esta propuesta, las posibilidades de incumplimiento - $-\mathrm{y}$, en consecuencia, acudir a los tribunales- se reducen prácticamente a cero por varias razones, como que:

- El acuerdo se valida y ejecuta de forma automática.

- Todas las operaciones derivadas de ese acuerdo son siempre visibles para las partes. 
- No puede ser cambiado o alterado de ninguna manera, sino que se ejecutará al pie de la letra sin presentar alteraciones o modificaciones en su contenido.

- Las condiciones que son preprogramadas en el acuerdo dan mayor certeza jurídica a las partes.

- Se eliminan o reducen en forma considerable los costos y el tiempo, al no tener que acudir a un tribunal para solicitar la ejecución forzosa.

- Habría constante supervisión, tanto por parte de la institución mediadora como por el tribunal competente.

\section{Retos de los contratos inteligentes para el cumplimiento y ejecución de acuerdos de transacción comerciales derivados de la mediación internacional}

Esta propuesta tiene muchas aristas que deben ser tomadas en cuenta y que no se pueden ignorar. A continuación, se expondrán cinco de las más evidentes.

Primero, los efectos jurídicos de los contratos inteligentes han caído en una zona gris, en virtud de que no han sido discutidos como tales ni en términos generales ni en forma específica en los congresos o legislaturas de la mayoría de los Estados, debido a que tienen características que los hacen difíciles de regular o incluso de prohibir. ${ }^{17}$ Sin embargo, esto no los convierte en la «nada jurídica», ya que su sola existencia produce consecuencias jurídicas. Es importante destacar que la doctrina aún no ha aceptado de forma unánime su naturaleza contractual (Tasende, 2020), sino que hay quienes consideran que son meramente programas informáticos (Lim, Hsu y Levy, 2016: 21). Otros estiman que sí es un contrato, en tanto se satisfagan los requisitos mínimos bajo la ley aplicable. ${ }^{18}$

Siguiendo la segunda hipótesis, en sentido amplio, que puede afirmar que los contratos inteligentes incluso se pueden equipar con los contratos electrónicos, Caffera (2003: 134) sostiene: «Cuando medios electrónicos constituyen el instrumento para perfeccionar un contrato, o bien para cumplirlo, se habla de contrato electrónico». Por lo tanto, en principio no necesitarían una regulación especial; sin embargo, en sentido estricto esto es bastante discutible, ya que las categorías y conceptos legales no son cien por ciento equiparables (Ríos, 2019: 4). Lo cierto es que esta decisión de-

17. Cabe señalar que esto paulatinamente está cambiando, ya que, por ejemplo, en Estados Unidos, Arizona, Delaware y Tennessee han reconocido a los contratos inteligentes. Estos estados de la Unión Americana han establecido el concepto de contrato inteligente y reconocido su eficacia jurídica. También en la Unión Europea se está empezando a legislar en la materia.

18. «Cryptoassets, DLT and smart contracts: UK Jurisdiction Taskforce consultation», The Law Society, 9 de mayo de 2019, disponible en bit.ly/3iqOYUx. 
penderá de cada país. Con este panorama en mente, cabe la posibilidad de que algunas instituciones que presten servicios de mediación internacional no los consideren como una opción hasta que estén debidamente regulados.

Segundo, en virtud de que estos contratos son autoejecutables, inmutables, descentralizados y autoverificables, fácilmente se pueden diseñar para que sean inmunes al poder coercitivo de cualquier Estado. Las cadenas de bloques en donde se alojan estos contratos son por lo general seudónimas y sin permisos, lo que hace casi imposible prevenir prácticas ilegales. Aunado a lo anterior, en caso de que se cause un daño a través de estos contratos, ¿a quién se debe fincar responsabilidad? ¿A las partes que establecieron las cláusulas del contrato, a los desarrolladores de la cadena de bloques, a los programadores que escribieron el código, o a los mineros encargados de verificar y ejecutar el contrato inteligente? Además, podría darse una usurpación de la identidad de las partes, ya que ellos se identifican a través de una clave privada $y$, si esta resulta comprometida, cualquiera que posea esa clave puede realizar transacciones no autorizadas a nombre del titular de la cuenta. Así, lejos de simplificar, el proceso de ejecución del acuerdo de transacción se complicaría aún más.

Tercero, como se explicó, el ámbito de aplicación de los contratos inteligentes es limitado, ya que solo funcionan para acuerdos que se realizan a partir de un grupo de condiciones predefinidas que se programan en un código informático. Se debe tomar en cuenta la complejidad que implica el programar estos contratos, en el caso de que se tengan que incorporar conceptos subjetivos como buena fe, valoración de hechos o pruebas (Koulu, 2016). Los contratos en principio operan fuera del control de un operador determinado y se ejecutan de forma automatizada, por lo que el cumplimiento de la obligación no da lugar a la interpretación o modificación, lo cual puede ocasionar graves problemas a las partes.

Cuarto, otro de los problemas a los que se enfrentan estos contratos es que vulneran el denominado derecho al olvido o derecho a la cancelación, el cual en México, por ejemplo, es considerado un derecho humano y se encuentra consagrado en el artículo 16 constitucional, segundo párrafo, que señala: «Toda persona tienen derecho a la protección de sus datos personales, al acceso, rectificación y cancelación de los mismos». A su vez, la Ley Federal de Protección de Datos Personales en Posesión de los Particulares señala en su artículo 25: «El titular tendrá en todo momento el derecho a cancelar sus datos personales». Como ya se explicó, en virtud de que estos contratos se encuentran en una cadena de bloques, la cual tiene la característica de ser inmutable, es imposible modificar o eliminar los datos registrados. ${ }^{19}$

Quinto, lo más común es que los acuerdos impliquen una suma de dinero. Este

19. Además, conviene señalar que, en materia contractual, se deben conservar los registros contables, y la temporalidad varía de legislación en legislación. En el caso de los contratos inteligentes, estos permanecen por siempre. 
dinero forzosamente tiene que ser en criptomonedas, que son un medio de pago intangible, sin respaldo y por completo descentralizado. Esta forma de pago tiene muchas ventajas, ya que se trata de monedas globales que no están reguladas por ningún tipo de organización, son deflacionarias, sus transacciones son irreversibles, son transparentes y son rápidas, ya que no necesitan pasar por un banco central. Sin embargo, también tienen desventajas: la primera es que nacieron en la irregularidad y, se podría decir, en la ilegalidad. Existe muy poca normativa al respecto, la cual en términos generales todavía es inefectiva y superficial (Barroilhet, 2019: 30). Son muy inestables, suben y bajan de precio constantemente. Por último, si se pierde la clave privada encriptada para acceder a la billetera electrónica, se pierde todo el dinero y no hay forma de recuperarlo.

\section{Conclusión}

La cadena de bloques o blockchain «es una tecnología de base de datos distribuida y a prueba de manipulaciones, que puede usarse para almacenar cualquier tipo de datos, incluyendo transacciones financieras» (OCDE, 2017: 345). El blockchain tiene una característica muy interesante: no necesita ninguna autoridad central u operador intermediario para funcionar. El ejemplo más claro del uso de blockchain es la criptomoneda Bitcoin, una divisa virtual y sistema de pagos descentralizado que opera en todo el mundo, independiente de cualquier banco central. Sin embargo, la cadena de bloques tiene otras aplicaciones transversales muy interesantes, una de las cuales son los contratos inteligentes.

Según lo planteado, los contratos inteligentes, por sus características inherentes, impactan de forma directa al derecho internacional privado en muchos aspectos, pero en particular en el ámbito de solución de conflictos. Se propone a los contratos inteligentes como una herramienta para asegurar el cumplimiento efectivo de los acuerdos de transacción comercial derivados de la mediación internacional, a fin de evitar que las partes acudan a los tribunales para solicitar su cumplimiento coactivo. Esta propuesta busca aumentar la velocidad de la ejecución de acuerdos derivados de una mediación internacional, lo cual debería traer como consecuencia el incremento de convenios con imposibilidad de incumplimiento, por lo que el riesgo de acudir a los tribunales o jueces sería casi nulo.

Por supuesto, las partes tendrían que ser informadas sobre las ventajas y desventajas de los contratos inteligentes; concordar desde un principio en que, de llegar a un acuerdo, este se plasmará en un contrato inteligente, con todas las implicaciones que esto conlleva (Vilalta, 2013: 54); y se debería garantizar que las cláusulas que se programen en el código binario estén dentro de los límites de la ley aplicable.

Ahora bien, al ser una tecnología de reciente creación, aunado a que los operadores jurídicos tienen una posición muy conservadora cuando se trata de innovaciones 
tecnológicas, los contratos inteligentes presentan muchos retos que se deben tomar en cuenta. De implementarse esta propuesta, en un inicio lo más conveniente será que tanto el juez competente como el mediador sean autorizados por las partes para intervenir digitalmente en la ejecución del contrato inteligente, en calidad de oráculos, a fin de darle certeza jurídica al proceso y garantizar derechos fundamentales.

\section{Referencias}

Barroilhet, Agustín (2019). «Criptomonedas, economía y derecho». Revista Chilena de Derecho y Tecnología, 8 (1): 29-67. DOI: 10.5354/0719-2584.2019.51584.

Bourque, Samuel y Sara Fung Ling Tsui (2014). «A lawyer's introduction to smart contracts». En Dušan Staněk (compilador), Scientia Nobilitat reviewed legal studies (pp 4-23). Varsovia: Aneta Tyc.

Bonnecase, Julien (1945). Elementos de derecho civil. Tomo 2. Ciudad de México: Cárdenas.

Caffera, Gerardo (2003). «Formación del contrato electrónico». En Comercio electrónico, análisis multidisciplinario (pp. 133-159). Buenos Aires: Julio César Faira Editor.

CASTRILLÓn, Víctor y Anahiby Becerril (2015). Contratación electrónica civil internacional. Ciudad de México: Porrúa.

Champagne, Phil (2014). El libro de Satoshi. Disponible en bit.ly/3z7kX1K.

CNUDMI, Comisión de Naciones Unidas para el Derecho Mercantil (2015). Solución de controversias comerciales: Ejecutabildad de los acuerdos de transacción derivados de procedimientos internacionales de conciliación/mediación. Nueva York: Naciones Unidas Publishing.

De Miguel, Pedro (2015). Derecho privado de internet. 5. a edición. Pamplona.

De Filippi, Primavera y Aaron Wright (2018). Blockchain and the law: The rule of code. Cambridge: Harvard University Press.

GonZÁlez, Nuria (2014). «Un acercamiento al acceso a la justicia a través de la mediación como medio alterno de solución de conflictos». En Sin derechos, exclusión y discriminación en el México actual (pp. 103-139). Ciudad de México: IIJ-UNAM.

-. (2020). «2020, el año de la Mediación en México: Iniciativa de Ley General y Convenio de Singapur. Revisemos y armonicemos en beneficio de los justiciables». En Opiniones técnicas sobre temas de relevancia nacional (pp. 9-25). Ciudad de México: IIJ-UNAM.

Koulu, Rikka (2016). «Blockchain and online dispute resolution: Smart contracts as an alternative to enforcement». Scripted, 13 (1): 40-69. DOI: 10.2966/scrip.130116.41.

LIM, Byungkwon, Emilie Hsu y Aaron Levy (2016). «In two recent orders, CFTC holds that Bitcoins are commodities». Intellectual Property \& Technology Law Journal, 28 (3): 19-23. 
LóPEz VAllés, Sara y Carlos Mauricio López Cárdenas (2014). «Aproximación a la regulación de la mediación en el derecho internacional privado y el derecho europeo». Revista de Derecho Privado, 51: 1-29. DOI: 10.15425/redepriv.51.2014.10.

Nofer, Michael, Peter Gomber, Oliver Hinz y Dirk Schiereck (2017). «Blockchain». Business \& Information Systems Engineering, 59 (7): 183-187. DOI: 10.1007/ s12599-017-0467-3.

ObServatorio Europeo de Blockchain (2019). Legal and REgUlatory fRaMEWORK OF BLOCKCHAIN AND SMART CONTRACTS. BRUSELAS: COMISIÓN EUROPEA.

OCDE, Organización para la Cooperación y Desarrollo Económicos (2017). Perspectivas de la OCDE sobre la economía digital 2017. Ciudad de México: OECD Publishing. DOI: 10.1787/9789264302211-es.

Padilla SánChez, Jorge Alberto (2020). «Blockchain y contratos inteligentes: Aproximación a sus problemáticas y retos jurídicos». Revista de Derecho Privado, 39: 175-201. DOI: 10.18601/01234366.n39.08.

Pereznieto, Leonel y Jorge Silva (2007). Derecho internacional privado: Parte especial. Ciudad de México: Oxford University Press.

Ríos, Yolanda (2019). «La tutela del consumidor en la "contratación inteligente": Los smart contracts y la blockchain como paradigma de la Cuarta Revolución Industrial». Revista de Consumo y Empresa, 9: 1-12. Disponible en bit.ly/3couUhM.

Smart Contracts Alliance (2016). Smart contracts: 12 use cases for business \& beyond. Washington, D. C.: Chamber of Digital Commerce.

SKLAROFF, Jeremy (2017). «Smart contracts and the cost of inflexibility». University of Pennsylvania Law Review, 166 (1): 263-303. Disponible en bit.ly/3cHTAC3.

STRONG, S. I. (2014). «Use and perception of international commercial mediation and conciliation: A preliminary report on issues relating to the proposed UNCITRAL Convention on International Commercial Mediation and Conciliation». University of Missouri School of Law Legal Studies Research Paper 2014-28. Disponible en bit.ly/3iCoPPJ.

Sultan, Karim, Umar Ruhi y Rubina Lakhani (2018). «Conceptualizing blockchains: Characteristics \& applications». En 11th IADIS International Conference Information Systems (pp. 49-57). Disponible en arxiv.org/abs/1806.03693.

Swan, Melanie. (2015). Blockchain: Blueprint for a new economy. Sebastopol: O'Reilly Media.

TAsende, Ignacio Jorge (2020). «Reflexiones sobre la nueva Convención de las Naciones Unidas sobre los Acuerdos de Transacción Internacionales resultantes de la mediación (Convención de Singapur)». Revista de Derecho de la Universidad de Montevideo, 19 (37): 71-91. Disponible en bit.ly/3cr4pIl.

Vilalta, Esther (2013). Mediación y arbitraje. Barcelona: Aranzadi. 


\section{Sobre los autores}

Wendolyne Nava GonzÁlez es abogada. Profesora e investigadora de tiempo completo en la Universidad Autónoma de Ciudad Juárez, México. Doctora en Derecho con especialidad en Derecho Internacional Privado por la Universidad de Barcelona, España. Miembro del Sistema Nacional de Investigadores de Conacyt, nivel 1. Editora de la Revista Especializa en Investigación Jurídica. Miembro de la Academia Mexicana de Derecho Internacional Privado y Comparado. Su correo electrónico es wendolyne.nava@uacj.mx. (D) https://orcid.org/oooo-0003-0711-0598.

Víctor Manuel Morales Rocha es ingeniero en sistemas computacionales. Profesor e investigador de tiempo completo en el Instituto de Ingeniería y tecnología de la Universidad Autónoma de Ciudad Juárez, México. Coordinador del Laboratorio Nacional de Tecnologías de información sede UACJ. Investigador asociado en el Centro de Inteligencia Artificial del estado de Chihuahua. Cursó sus estudios de doctorado en la Universidad Politécnica de Cataluña, España. Su correo electrónico es victor.morales@uacj.mx. (D) https://orcid.org/oooo-0oo1-8213-400X. 


\title{
REVISTA CHILENA DE DERECHO Y TECNOLOGÍA
}

La Revista de Chilena de Derecho y Tecnología es una publicación académica semestral del Centro de Estudios en Derecho Informático de la Facultad de Derecho de la Universidad de Chile, que tiene por objeto difundir en la comunidad jurídica los elementos necesarios para analizar y comprender los alcances y efectos que el desarrollo tecnológico y cultural han producido en la sociedad, especialmente su impacto en la ciencia jurídica.

\author{
EDITOR GENERAL \\ Daniel Álvarez Valenzuela \\ (dalvarez@derecho.uchile.cl) \\ SITIO WEB \\ rchdt.uchile.cl \\ CORREO ELECTRÓNICO \\ rchdt@derecho.uchile.cl \\ LICENCIA DE ESTE ARTÍCULO \\ Creative Commons Atribución Compartir Igual 4.o Internacional
}

\begin{abstract}
La edición de textos, el diseño editorial
y la conversión a formatos electrónicos de este artículo

estuvieron a cargo de Tipográfica

(www.tipografica.io).
\end{abstract}

